\title{
Bürgergeld und die Zukunft des Sozialstaats
}

Die Ende 2021 neu gewählte deutsche Bundesregierung plant eine Reform von Hartz IV hin zu einem Bürgergeld. Der Koalitionsvertrag der Ampel sieht dabei vor, dass es ein Sanktionsmoratorium geben wird, die Anrechnung des Zuverdienstes reduziert und auf die Überprüfung von Vermögen sowie Angemessenheit der Wohnung in den ersten zwei Jahren verzichtet wird. Hervorheben lässt sich zudem, dass zwar auf Sanktionen nicht grundsätzlich verzichtet, aber der Vermittlungsvorrang abgeschafft werden soll. Kompetenzen und Entwicklungsbedarfe der Erwerbsfähigen sollen besser ermittelt und Weiterbildung wie Qualifizierung verbessert sowie durch ein Weiterbildungsgeld auch finanziell unterstützt werden. Die Jobcenter sollen mehr Gestaltungsspielraum bekommen. Darin wird das Bemühen deutlich, die Arbeitsförderung bei erwerbsfähigen Grundsicherungsempfänger:innen in nachhaltige und bessere Jobs zu stärken. Damit würden sich die Bedingungen der Grundsicherung für Arbeitssuchende (SGB II) an wesentlichen Stellen ändern.

Wie ist diese Reform systematisch einzuordnen? Handelt es sich dabei um ein „Grundeinkommen light" oder nur um eine bessere Sozialhilfe? Welche Perspektiven für die Zukunft des Sozialstaats bildet diese Reform und welche Fragen bleiben kurzfristig noch offen? Welche weiteren Anpassungen sollte es im Zuge der Reform geben, beispielsweise bei der Grundsicherung für Erwerbsminderung oder im Alter (SGB XII) ( - wobei die Grundsicherung im Alter für langjährig Versicherte mit der neuen Grundrente bzw. präziser dem Grundrentenzuschlag ohnehin überflüssig werden könnte)? Ist die derzeitige Höhe der Regelsätze angemessen und sind die im Koalitionsvertrag angelegten Reformen weitreichend genug? Wie soll das Ziel einer geringeren Grenzbelastung erreicht werden? Zur Konkretisierung sieht der Koalitionsvertrag vor, eine unabhängige Kommission mit der Entwicklung eines Reformmodells zu beauftragen.

Wir wollen diesen Fragen vor dem Hintergrund einer seit Jahrzehnten geführten wissenschaftlichen und politischen Diskussion um die Zukunft des Sozialstaats (Strengmann-Kuhn, 2005; Opielka, 2008) nachgehen und dabei auf veröffentlichte Befunde aus einem für diese Diskussion einschlägigen Projekt der Zukunftsforschung

(C) Der/die Autor:in 2022. Open Access: Dieser Artikel wird unter der Creative Commons Namensnennung 4.0 International Lizenz veröffentlicht (creativecommons.org/licenses/by/4.0/deed.de).

Open Access wird durch die ZBW - Leibniz-Informationszentrum Wirtschaft gefördert.
Bezug nehmen, auf das von der sogenannten Jamaikakoalition in Kiel mit dem Koalitionsvertrag in 2017 initiierte „Zukunftslabor Schleswig-Holstein“, dessen Ergebnisse ursprünglich im Frühjahr 2021 vorgelegt werden sollten. ${ }^{1}$ Das Projekt soll in einem wissenschaftlich angeleiteten öffentlichen Diskurs eine Vision für die Zukunft und Nachhaltigkeit der sozialen Sicherung entwickeln und dabei vor allem auch die Rolle eines Grundeinkommens bzw. Bürgergelds prüfen: Durch eine Bestandsanalyse bestehender sozialer Sicherungssysteme vor dem Hintergrund der demografischen Entwicklung und der fortschreitenden Digitalisierung sowie einer Prognose zu den Auswirkungen auf die sozialen Sicherungssysteme, durch die Darstellung verschiedener alternativer Modelle sowie der Entwicklungsmöglichkeit bestehender sozialer Sicherungssysteme und durch die Aufbereitung und Auswertung der Erfahrungen auf Bundes- und EU-Ebene (Opielka, 2019).

Im Kontext des Zukunftslabors wurde ein differenziertes Modell aus vier Reformszenarien entwickelt, die durch das DIW auf ihre Auswirkungen auf die Fiskal- und Arbeitsmarktpolitik simuliert werden sollten (Opielka und Peter, 2020). Alle vier Reformszenarien beinhalten ein mehr oder weniger weitreichendes Grundeinkommen sowie damit systematisch korrespondierende Reformideen zur Finanzierung der Kranken- und Pflegeversicherung: 1) Bürgergeld 2) Grundeinkommen 3) Sozialversicherung

1 Sie hätten dadurch in die Pläne der Ampel- oder jeder anderen Regierungskoalition einbezogen werden können. Die Autoren waren als Projektleiter und Berater in das Projekt einbezogen: www.zlabsh.de Das Schicksal des Zukunftslabors wird derzeit vor dem Landgericht Flensburg verhandelt. Es erscheint nicht ausgeschlossen, dass das Projekt nach der Landtagswahl Schleswig-Holstein im Mai 2022, möglicherweise in einer anderen Regierungskoalition, fortgeführt wird.

Prof. Dr. habil. Michael Opielka ist Wissenschaftlicher Leiter und Geschäftsführer des ISÖ - Institut für Sozialökologie gGmbH in Siegburg und Professor für Sozialpolitik an der Ernst-Abbe-Hochschule Jena.

Dr. habil. Wolfgang Strengmann-Kuhn ist Senior Fellow im ISÖ - Institut für Sozialökologie gGmbH und Obmann der Bundestagsfraktion Bündnis 90/Die Grünen im Ausschuss für Arbeit und Soziales. 
Tabelle 1

Vier Reformszenarien zur Zukunft des Sozialstaats

\section{Bürgergeld \\ (mit Bürgerpauschale)}

Das Bürgergeld in Form einer „Negativen Einkommensteuer" dient vor allem der Unterstützung der Arbeitsmarkt- und Leistungsmotivation in den unteren Arbeitsmarktsegmenten. Die Bürgerpauschale (Kopfpauschale) für Gesundheit und Pflege dient der Förderung des Wettbewerbs zwischen gesetzlichen und privaten Kranken-/Pflegekassen.

\section{Grundeinkommen \\ (mit steuerfinanziertem Gesund- \\ heits- und Pflegesystem)}

Das Grundeinkommen in Form einer "Sozialdividende" steht jeder/m legalen Einwohner:in monatlich zu und unterliegt (analog zum „Primäreinkommen") der Einkommensteuer- und Sozialversicherungsbeitragspflicht. Das Gesundheits-/ Pflegesystem wird vollständig aus Steuermitteln finanziert.
Sozialversicherung

(mit Grundsicherung bzw. Garantiesicherung)

Beitragsfinanzierte, lebensstandardsichernde („Bismarcksche") Sozialversicherung mit "Sockelung durch bedarfsorientierte Grundsicherung (,,Garantiesicherung“). Gesundheits-/Pflegeversicherung wie bisher im gegliederten System (GKV, PKV, Beihilfe).
Bürgerversicherung

(mit Grundeinkommensversicherung)

Grundeinkommensversicherung nach dem Modell der Schweizer AHV in allen Risikolagen für Geldleistungen (Alter, Arbeitslosigkeit, Elternschaft, Krankheit, Behinderung, Kindheit, Ausbildung) und für den Risikobereich Gesundheit/ Pflege.

Quellen: https://www.isoe.org/aktuelles/news/delphi-befragung-im-zukunftslabor-gestartet-ihr-expertinnen-wissen-ist-gefragt-beteiligen-sie-sich/ (1. Februar 2022); Hutflesz und Opielka (2020, 63 ff.).

(mit Grundsicherung oder Garantiesicherung) 4) Bürgerversicherung (vgl. Tabelle 1, detailliert Hutflesz und Opielka, 2020, 63 ff.). ${ }^{2}$ Die vier Reformszenarien lassen sich mit den aus der politischen Soziologie bekannten vier Typen des Wohlfahrtsregimes in Verbindung bringen, die erstmals von Gøsta Esping-Andersen formuliert wurden: liberal, sozialdemokratisch (sozialistisch), konservativ und garantistisch (Opielka, 2008, 2017). Im Folgenden werden wir uns auf die vier Szenarien zur Reform der Einkommenssicherung konzentrieren, die Reformoptionen für die Kranken- und Pflegeversicherung können hier nur kurz berührt werden.

Es fällt auf, dass der Begriff „Bürgergeld“ im Szenariomodell anders verwendet wird als im Koalitionsvertrag der Ampel. Dahinter steht ein bemerkenswerter, historisch interessanter semantischer Wandel. Der Begriff „Bürgergeld“ wurde erstmals von den liberalen Ökonomen Wolfram Engels und Joachim Mitschke in den 1970er Jahren als Synonym für die Umsetzung eines Grundeinkommens als „Negative Einkommensteuer" verwendet. Er setzte sich in der deutschen Diskussion weitgehend durch, beispielsweise mit der „Kommission Bürgergeld/Negative Einkommensteuer KoBüNE“ der FDP, die 2004-5 von Andreas Pinkwart geleitet wurde (KoBüNE und Pinkwart, 2005). Stärker beachtet wurde das Grundeinkommensmodell „Solidarisches Bürgergeld“ des damaligen Thüringer Ministerpräsidenten Dieter Althaus, das Mitte der 2000 er Jahre vorgestellt, auf seine Finanzierbarkeit berechnet wurde (Opielka und Strengmann-Kuhn, 2007) und ebenso zu einer Kommission bei einem Parteivorstand führte, diesmal der CDU. Überraschend nutzte die damalige SPD-Parteivorsitzende Andrea Nahles den bislang

2 Die Simulation von Reformszenario 1 liegt für den Einkommensteil „Bürgergeld“ in einer ersten Fassung vor (Bach et al., 2020). liberal-konservativ verorteten Begriff „Bürgergeld“ im Jahr 2019 für eine parteiprogrammatische Revision der von Bundeskanzler Gerhard Schröder und seinem Chef des Bundeskanzleramts, Frank-Walter Steinmeier, in den Jahren 2003-4 initiierten „Agenda 2010“ mit der folgenreichen Reform „Hartz IV“.

Noch vor der SPD haben Bündnis 90/Die Grünen, das Ziel einer Überwindung von Hartz IV propagiert. Vor dem Hintergrund der jahrzehntelangen Diskussion der Grünen zum Grundeinkommen (Opielka und Strengmann-Kuhn, 2021) versuchte Robert Habeck im Jahr 2018 die Kontroverse um das Grundeinkommen mit dem Vorschlag einer „Garantiesicherung“ zu befrieden (Habeck, 2018). ${ }^{3}$ Dieser Vorschlag wurde anschließend von der grünen Bundestagsfraktion (Bundestagsfraktion Bündnis 90/Die Grünen, 2020) konkretisiert, weiterentwickelt und schließlich von der Partei ins Wahlprogramm übernommen. Die Garantiesicherung ist dabei kein Grundeinkommen, sondern hat wie der Nahles/SPD-Vorschlag „Bürgergeld“ das Ziel, innerhalb der strukturell zunächst unveränderten, korporatistisch gegliederten und insoweit konservativen deutschen Sozialstaatsarchitektur inkrementalistisch, Schritt für Schritt, die bedarfsorientierten Fürsorgeleistungen des Typus Sozialhilfe bürgerrechtlich zu modernisieren, gehört also nach der obigen Systematik in die Kategorie „Grundsicherung“. Die FDP suchte über ihre Friedrich-Naumann-Stiftung mit einem ifo-Gutachten 2019 ein Update ihrer Bürgergeldkonzeption, weg von der Negativsteuer hin zu einer Grundsicherung (Blömer und Peichl, 2019). Dass die drei Ampelparteien SPD, FDP und Bündnis 90/Die Grünen zur Überraschung der politischen

3 Siehe dazu auch den Kommentar von Michael Opielka zum Vorschlag von Robert Habeck: https://www.isoe.org/aktuelles/blog/grundeinkommen-light-zu-robert-habecks-garantiesicherung/ (1. Februar 2022). 
und wissenschaftlichen Öffentlichkeit in den Koalitionsverhandlungen ein Bürgergeld lancierten, erscheint daher durchaus naheliegend, obwohl es sich eigentlich nicht um ein Bürgergeld im Sinne einer Negativen Einkommensteuer handelt. Sowohl bei FDP als auch Grünen ist aber in den Partei- bzw. Wahlprogrammen vorgesehen, das Bürgergeld bzw. die Garantiesicherung schrittweise in das Steuersystem zu integrieren.

Das „Bürgergeld“ der Ampel gehört also in das konservative Wohlfahrtsstaatsregime der oben dargestellten Systematik, hat aber nach unserem Dafürhalten das Potenzial für eine Weiterentwicklung in Richtung der anderen Typen einer Mindestsicherung bzw. eines Grundeinkommens. Wir wollen anhand von vier Problemstellungen noch offene Probleme sowie die Chancen und Perspektiven dieses sozialpolitischen Pragmatismus diskutieren: a) die Niveaufrage der Grundsicherung, b) das Anrechnungsproblem sonstiger Einkommen, c) die Bezüge zu Grundrente und Bürgerversicherung und d) die Kindergrundsicherung.

\section{Niveaufrage}

Alle relevanten sozialpolitischen Akteur:innen fordern seit Jahren eine wirksame und strukturell abgesicherte Anhebung des Grundsicherungsniveaus der Regelsätze, um die Verfestigung materieller Armut zu verhindern. Hier ist nicht der Raum, um die vielfältigen Implikationen dieser Diskussion zu würdigen, vom Bemessungsmodus (Statistikmodell vs. Warenkorb) über die Bezugseinheit (Haushalt, Alleinerziehendenstatus), dem Verhältnis zu anderen Minima (Pfändungsfreigrenze, Steuerfreibetrag), der Einbettung in andere Transferleistungen (Wohngeld, Kostenfreiheit öffentlicher Dienstleistungen und so weiter) bis hin zur nachhaltigkeitspolitischen Frage, was und wieviel „der Mensch“ eigentlich braucht. Irene Becker hat ein durchaus plausibles Modell entwickelt, das zu einem $46 \%$ höheren Regelsatz führt (Becker, 2022). Der Koalitionsvertrag hält sich in Sachen Niveau zurück. Lediglich für Kinder ist eine Anhebung der Leistungen vorgesehen. Wir gehen davon aus, dass die Niveaufrage erst in der nächsten Legislaturperiode seriös behandelt werden kann. ${ }^{4}$ Möglicherweise erleichtern die mit dem Bürgergeld verbundenen Strukturreformen auch eine großzügigere Antwort auf die Niveaufrage.

Kurzfristig wirkt sich die überinflationäre Verteuerung der Energiekosten, vor allem der fossilen Energien, gerade in den untersten und erheblich von Transferleistungen lebenden Einkommensgruppen dramatisch aus. Nicht

4 Die nächste reguläre Neuberechnung zur Anhebung der Regelsätze 2026 durch das Regelbedarfsermittlungsgesetz (RBEG) steht im Jahr 2025 an. genug damit, auch die überinflationäre Steigerung der (Kalt-)Mieten und Immobilienpreise führt vor allem in Ballungsräumen zu einer Strapazierung sozialstaatlicher Instrumente wie Wohngeld oder Wohnungsbauförderung für Schwellenhaushalte. Einmalzahlungen helfen den Betroffenen über erste Notlagen, reichen aber häufig schon von der Höhe nicht aus, den Kaufkraftverlust auszugleichen. Deswegen braucht es systematische Lösungen, zumal mittelfristig durch die wünschenswerte Internalisierung ökologischer Kosten mit weiteren Preissteigerungen zu rechnen ist, die Menschen mit geringen Einkommen stark belasten. Ohne sozialpolitische Flankierung könnte die Beschleunigung der Klimapolitik das Abkoppeln armutsnaher Haushalte von der Wohlstandsentwicklung gravierend zuspitzen und damit zur Delegitimierung der Klimapolitik wesentlich beitragen („Gelbwesten“). Das geplante Klimageld im Koalitionsvertrag kann dazu einen wichtigen Beitrag leisten, eine Konkretisierung steht allerdings aus und sollte nicht auf die lange Bank geschoben werden. Ein als jährliche (oder monatliche) Pro-Kopf-Pauschale an alle Bürger:innen ausgezahltes Klimageld bzw. Energiegeld als Bestandteil eines in Richtung Grundeinkommen erweiterten Bürgergelds könnte dabei auf Erfahrungen der Schweiz zurückgreifen. Dort wird es über die Krankenversicherung ausgezahlt. ${ }^{5}$ Solange es in Deutschland keine Bürgerversicherung gibt, ist das aber nicht eins zu eins übertragbar. Eine Alternative wäre eine Auszahlung über die Finanzämter, wenn das Bürgergeld in das Steuersystem integriert würde. Ein um ein Klimageld ergänztes Bürgergeld dürfte die Akzeptanz sowohl für den Klimaschutz wie für ein Grundeinkommen steigern.

\section{Anrechnungsproblem}

Die Ampelkoalition erhofft sich durch niedrigere Anrechnungsraten für Erwerbseinkommen mit der BürgergeldReform eine Reduzierung der „Armutsfalle“ und eine Dynamisierung der Arbeitsmärkte. Sie hat das Ziel, dass Erwerbsarbeit auch im unteren Einkommenssegment nicht prohibitiv belastet werden soll. Zu dieser Frage soll eine unabhängige Kommission eingerichtet werden, die Vorschläge zur Reduzierung der Grenzbelastung auch im Zusammenspiel mit anderen Sozialleistungen vorlegen soll. Eine deutliche Erhöhung der Leistungen sowie die Senkung der Transferentzugsraten würden die Zahl der Berechtigten deutlich erhöhen und hohe fiskalische Kosten auslösen. Außerdem gibt es unterschiedliche Effekte für das Arbeitsangebot: Einerseits ergeben sich

5 Seit 2008 erhebt in der Schweiz der Bund auf fossile Brennstoffe wie Heizöl oder Erdgas eine $\mathrm{CO}_{2}$-Abgabe. Das Bundesamt für Umwelt (BAFU) sorgt für die Verteilung der Umweltabgaben an alle Versicherten, und zwar via Krankenversicherer. Im Jahr 2021 wurden 87 Franken aus den Erträgen der Umweltabgaben $\left(\mathrm{CO}_{2}\right.$-Abgabe und VOCAbgabe) zurückgezahlt. 
durch die geringere Grenzbelastung gerade für bisher Erwerbslose oder geringfügig Beschäftigte positive Arbeitsangebotseffekte, andererseits kann die Leistungsausweitung negative Arbeitsanreize auslösen, die wiederum durch die Mindestlohnerhöhung und generell steigende Löhne zumindest abgeschwächt werden könnten. Neben den fiskalischen Wirkungen und den komplexen Arbeitsangebotswirkungen sollte aber auch berücksichtigt werden, dass durch eine Verringerung der Transferentzugsraten zunehmend Erwerbstätige Anspruch auf Bürgergeld erhalten würden. Eine Abwicklung über die Jobcenter erscheint für diese Gruppe nicht angebracht. Außerdem zahlt diese Gruppe in der Regel Einkommensteuern. Die Konsequenz könnte eine stärkere Integration von Steuer- und Transfersystem sein, also eine Entwicklung in Richtung Negative Einkommensteuer und damit dem "Bürgergeld“ in der Zukunftslabor-Systematik. Das ist nicht unmöglich und wurde in vielen Varianten vorgedacht (Blömer und Peichl, 2018; Buhlmann, Kolb und Siegloch, 2020).

\section{Grundrente und Bürgerversicherung}

Würde das Ampel-Bürgergeld in Richtung Bürgerversicherung, beispielsweise in Form einer "Grundeinkommensversicherung“ (Opielka, 2008) entwickelt, kämen die großen programmatischen Linien von SPD und Bündnis 90/Die Grünen stärker zur Geltung. Die "Grundrente" der letzten Großen Koalition, faktisch ein "Grundrentenzuschlag"6, hat die Grundsicherung für Rentner:innen der Gesetzlichen Rentenversicherung (GRV) für lange gesetzlich Versicherte zunehmend, allerdings nicht vollständig ersetzt. Nicht oder nicht ausreichend lang Versicherte sind aber auch weiterhin auf Grundsicherung im Alter angewiesen. Insoweit sollte auch bei der Bürgergeld-Reform der Blick auf die Statik der Alterssicherungssysteme gelegt werden, sowohl in Bezug auf die Grundsicherung im SGB XII als auch die Weiterentwicklung der GRV zu einer Bürgerversicherung, die idealerweise allen Versicherten eine Grundrente garantiert. Für das Ziel Bürgerversicherung spricht empirisch, dass in den letzten Jahrzehnten und durch die Coronapandemie erneut beschleunigt der Anteil der Zuschüsse aus dem Bundeshaushalt an die Sozialversicherung gewaltig stieg. ${ }^{7}$ Die enormen Steuerleistungen an die Sozialversicherungen zeigen: Sie wandeln sich zuneh-

6 So nennt es unterdessen auch die DRV: https://www.deutsche-rentenversicherung.de/DRV/DE/Rente/Grundrente/grundrente_node. html (1. Februar 2022).

7 So beträgt im Jahr 2022 der Bundeszuschuss an die GRV 108 Mrd. Euro (BMF, 2021, 24), an den Gesundheitsfonds der GKV 21,5 Mrd. und an die Pflegeversicherung 1 Mrd. Euro (BMF, 2021, 27 f.). Die Bundeszuschüsse an die Sozialversicherungen belaufen sich damit auf annähernd $30 \%$ des Bundeshaushalts. Hinzu kommen die Pensionskosten für Beamte allein für den Bund mit 9,4 Mrd. Euro in 2022 (BMF, 2021, 23). mend in Bürgerversicherungen, doch halten am Mythos der Erwerbsbezogenheit fest. Eine Bürgergeld-Reform, die auch die Finanzierung und Konstruktion der Sozialversicherungen mit in den Blick nimmt, könnte mit solchen Mythen und damit Illusionen aufräumen.

\section{Kindergrundsicherung}

Die Einführung einer Kindergrundsicherung gehört zu weiteren Schwerpunkten des Ampelkoalitionsvertrags. Sie hängt für alle Haushalte mit Kindern mit der Bürgergeld-Reform zusammen. Wir sehen eine Brücke in Richtung Grundeinkommen über den „Garantiebetrag“, der laut Koalitionsvertrag „perspektivisch so hoch“ liegen soll, wie die maximale Steuerersparnis durch die Kinderfreibeträge, also derzeit 314,55 Euro ${ }^{8}$. Das erinnert an die geltende Regelung im Kindergeld durch die Wahloption Kinderfreibetrag: Faktisch ist das Kindergeld dadurch in das Einkommensteuersystem als partielles Grundeinkommen für Kinder integriert, es umfasst je nach Alter heute zwischen $58 \%$ und $76 \%$ des Regelsatzes für Kinder. Dieser Betrag soll perspektivisch beim „Garantiebetrag“ der Kindergrundsicherung deutlich höher sein. Außerdem soll dieser "Garantiebetrag“ für volljährige Kinder anders als das heutige Kindergeld direkt an diese und nicht mehr an die Eltern ausgezahlt werden, hätte also für diese Personengruppe, bei der es sich insbesondere um Studierende handelt, den Charakter eines partiellen Grundeinkommens. Eine solche Lösung ist auch für das Bürgergeld der Nicht-mehr-Kinder denkbar. Alexander Spermann schlug mit dem „Basisgeld“ ein partielles Grundeinkommen in Höhe des Regelsatzes vor (Spermann, 2019). Die Kindergrundsicherung könnte die sozialpolitische Entwicklung in der Zukunftslabor-Systematik damit in Richtung Grundeinkommen führen.

\section{Ergebnis}

Vor dem Hintergrund einer Systematik der Zukunftsperspektiven des Sozialstaats, wie sie zuletzt im „Zukunftslabor Schleswig-Holstein" unsererseits vorgeschlagen wurde, erscheint das „Bürgergeld“ im Koalitionsvertrag der Ampelregierung auf den ersten Blick konservativ und bestandswahrend und weit entfernt von den mutigen Grundeinkommensexperimenten wie zuletzt in Finnland (Merrill et al., 2022). Unsere Diskussion zeigt jedoch, dass die Synthese der unterschiedlichen Herkunftslinien das Potenzial zu einer inkrementalistischen, aber nachhaltigen

8 Die Kinderfreibeträge bestehen aus dem sächlichen Existenzminimum in Höhe von 5.460 Euro und dem sogenannten BEA-Freibetrag für Betreuungs-, Erziehungs- und Ausbildungsbedarf in Höhe von 2.928 Euro. Beim Spitzensteuersatz von $45 \%$ ergibt sich dann eine Ersparnis von 314,55 Euro. Zur Problematik des u-förmigen Verlaufs der aktuellen Kindergrundsicherung siehe Breuer (2018). 
Reform der Sozialpolitik in Richtung Bürgerversicherung und Grundeinkommen birgt. Die Ängste der politischen Eliten sind hierbei weitaus größer als die Erwartungen der Bürger:innen, genau in diese Richtung hin zu gestalten. Die angedeutete Integration mit anderen Sozialleistungen (Wohngeld, Kindergrundsicherung) und vor allem ein einfacherer und digitaler Zugang zu den Leistungen dürften die bisher hohe Nichtinanspruchnahme der Grundsicherung verringern und das Bürgergeld in Richtung Grundeinkommen für alle Sozialbürger:innen bewegen.

\section{Literatur}

Bach, S., P. Brock und L. Kampfmann (2020), Mikrosimulationsanalysen zu den Reformszenarien (Bürgergeld). Erste Ergebnisse zur Implementation, Ms., DIW.

Becker, I. (2022), Sicherung des Existenzminimums mit Regelleistungen, in: F. Blank et al. (Hrsg.), Grundsicherung weiterdenken, 61-84, transcript.

Blömer, M. und A. Peichl (2018), Ein Garantieeinkommen für alle, ifo Forschungsbericht, 97.

Blömer, M. und A. Peichl (2019), Anreize für Erwerbstätige zum Austritt Algll - Liberales Bürgergeld, ifo, Friedrich-Naumann-Stiftung, ifo fb, 98, 2-2019.

Breuer, C. (2018), Ein Grundeinkommen für Kinder, Wirtschaftsdienst, 7(98), 481-488, https://www.wirtschaftsdienst.eu/inhalt/jahr/2018/heft/7/ beitrag/ein-grundeinkommen-fuer-kinder.html (3. Februar 2022).

Buhlmann, F., M. Kolb und S. Siegloch (2020), Einführung einer Garantiesicherung, Endbericht, Gutachten des ZEW im Auftrag der Bundestagsfraktion von Bündnis 90/Die Grünen.

Bundestagsfraktion Bündnis 90/Die Grünen (2020), Hartz IV überwinden - Garantiesicherung einführen. Fraktionsbeschluss vom 20.12.2020, https://www.gruene-bundestag.de/files/beschluesse/beschluss-garantiesicherung.pdf (1. Februar 2022).
BMF (2021), Finanzbericht 2022

Habeck, R. (2018), Anreiz statt Sanktionen, bedarfsgerecht und bedingungslos. Wie wir die Beschlusslagen der Partei umsetzen und ein Garantiesystem aufbauen. Ein Debattenbeitrag von Robert Habeck zum Grundsatzprogramm, https://www.gruene.de/artikel/anreiz-stattsanktionen-bedarfsgerecht-und-bedingungslos (1. Februar 2022).

Hutflesz, T. und M. Opielka (2020), Online-Delphi in der Zukunftsforschung zur Sozialpolitik, ISÖ-Text, 2020-3, BoD.

KoBüNE - Kommission Bürgergeld Negative Einkommensteuer und A. Pinkwart (2005), Das Liberale Bürgergeld: aktivierend, transparent und gerecht, FDP.

Merrill, R., C. Neves und B. Lain (Hrsg.) (2022), Basic Income Experiments. A Critical Examination of Their Goals, Contexts, and Methods, Palgrave Macmillan.

Opielka, M., und W. Strengmann-Kuhn (2007), Das solidarische Bürgergeld. Finanz- und sozialpolitische Analyse mit Empfehlungen zur Operationalisierung, Studie im Auftrag der Konrad-Adenauer-Stiftung (unter Mitarbeit von Bruno Kaltenborn), in M. Borchard (Hrsg.), Das Solidarische Bürgergeld. Analysen einer Reformidee, 13-141, Lucius \& Lucius.

Opielka, M. (2008), Sozialpolitik. Grundlagen und vergleichende Perspektiven, 2. Aufl., Rowohlt.

Opielka, M. (2017), Welche Zukunft hat der Sozialstaat? Eine Prognose, Deutscher Verein/Lambertus.

Opielka, M. (Hrsg.) (2019), Zukunftslabor Schleswig-Holstein. Demographie und Digitalisierung \#ZLabSH, ISÖ-Text, 2019-1, BoD.

Opielka, M. und S. Peter (2020), Zukunftslabor Schleswig-Holstein. Zukunftsszenarien und Reformszenarien, ISÖ-Text, 2020-1, BoD.

Opielka, M. und W. Strengmann-Kuhn (2021), The Green discussion on basic income in Germany - its development and current status, in Green European Foundation (Hrsg.), European Green Perspectives on Basic Income, Vol. II, August, 58-63, GEF.

Spermann, A. (2019), Basisgeld plus Steuergutschriften statt Hartz IV, Wirtschaftsdienst, 3(99), 181-188, https://www.wirtschaftsdienst.eu/ inhalt/jahr/2019/heft/3/beitrag/basisgeld-plus-steuergutschriftenstatt-hartz-iv.html (3. Februar 2022).

Strengmann-Kuhn, W. (Hrsg.) (2005), Das Prinzip Bürgerversicherung. Die Zukunft im Sozialstaat, Springer VS.

Title: Citizen's Income and the Future of the Welfare State

Abstract: The authors discuss the reform of basic security in the direction of a "citizen's income" in the coalition agreement of the new "traffic light coalition" at the federal level against the background of fundamental considerations on the future of the welfare state, including those from the "Schleswig-Holstein Future Laboratory". They see the reform of the citizen's income as an incrementalist step toward a basic income and discuss their thesis on four topics: a) the question of the level of the basic income, b) the problem of counting other incomes, c) the relationship to the basic pension and citizen's insurance, and d) the basic income for children. 